\title{
A Class of Modified Ratio Estimators Using Linear Combination of Quartile Deviation and Median of Auxiliary Variable Under Rank Set Sampling
}

\author{
M. Iqbal Jeelani, S.A.Mir, M.S.Pukhta* \\ Division of Agricultural Statistics, SKUAST-K, India \\ *Corresponding Author: mspukhta_67@yahoo.co.in
}

Copyright (C) 2014 Horizon Research Publishing All rights reserved.

\begin{abstract}
In this paper we suggest a new modified ratio estimator of population mean of the study variable using the linear combination of known values of Quartile deviation and Median of the auxiliary variable under Rank set sampling. Mean squared error up to the first degree of approximation are derived and compared with modified ratio estimators given by Kadilar and Chingi [1] based on simple random sampling. The proposed modified ratio estimators under rank set sampling perform better than the ratio estimators given by Kadilar. The simulated study has been carried out in support of the results.
\end{abstract}

Keywords Rank set sampling, Ratio type estimator, Simple random sampling, Mean square error, Quartile deviation, Median

\section{Introduction}

Most of the times in sample surveys, along with the variable of interest $Y$, information on auxiliary variable $X$, which is highly correlated with $Y$ is also collected. This information on auxiliary variable may well be utilized to obtain a more efficient estimator of population mean. Ratio method of estimation is one such example which utilizes the information on auxiliary variable $X$, which is positively correlated with the variable of interest $Y$, in order to improve the precision of the estimate of population mean. An alternative method to SRS called Ranked Set Sampling (RSS) was introduced to increase the efficiency of the estimation of population mean [2]. The method is useful when the variable of interest is very expensive or difficult to measure but it can be easily ranked at a negligible cost. There are cases in practical situation where the variable of interest $\mathrm{Y}$ is difficult to measure and to rank but a concomitant variable $\mathrm{X}$, which is highly correlated with $\mathrm{Y}$, can be easily ranked and be used for the ranking of the sampling units. Some extension is done by Swami [3] utilized both the rank and the measure of the concomitant variable and considered ratio estimation using RSS. The ratio estimation based on RSS is more efficient compared with the SRS ratio estimate.

\section{Ratio Estimation under Simple Random Sampling}

Let the variable of interest $\mathrm{Y}$ and the concomitant variable $\mathrm{X}$ is correlated with the coefficient of correlation $\rho$.The population ratio of these two variable is then $R=\mu_{y} / \mu_{x}$ or $R=\bar{Y} / \bar{X}$ and its estimator is $\hat{R}=\frac{\bar{y}}{\bar{x}}$, where $\mu_{y}$ and $\mu_{x}$ are the populations means of the variables $\mathrm{Y}$ and $\mathrm{X}$ respectively, $\bar{y}$ and $\bar{x}$ are the sample mean for $\mu_{x}$ and $\mu_{y}$ respectively. The ratio estimator is biased but the bias is negligible when the estimator is approximated using Taylor series expansion to the first degree, Cochran[4]. The approximated Variance of $\hat{R}$ is

$$
\begin{gathered}
\operatorname{Var}(\hat{R}) \cong \frac{R^{2}}{n}\left(V_{x}^{2}+V_{y}^{2}-2 \rho_{x} V_{x} V_{y}\right) \\
\text { Where, } V_{x}=\frac{\sigma_{x}}{\mu_{x}}, V_{y}=\frac{\sigma_{y}}{\mu_{y}} \text { and } \\
\rho_{x y}=\sum_{i=1}^{N}\left(x_{i}-\mu_{x}\right)\left(\mu_{i}-\mu_{y}\right) / N \sigma_{x} \sigma_{y}
\end{gathered}
$$

$\sigma_{x}$ and $\sigma_{y}$ are the standard deviations of the populations of the variables $\mathrm{X}$ and $\mathrm{Y}$, respectively.

Many ratio type estimators based on SRS have been proposed. Improvements in the precision of the estimator through the use of Ratio method of estimation are achieved by introducing a large number of modified Ratio estimators which utilizes the information on known values of Co-efficient of variation, Co-efficient of kurtosis, Co-efficient of Skewness etc. Some of the modified Ratio estimators in case of SRS given by Murthy [5], Cochran [4], Prasad [6], Sen [7], Singh and Tailor [8, 9], Singh et.al [10], Kadilar and Cingi [1, 11], Koyuncu and Kadilar [12], Yan and Tian [13], M Iqbal et al [14]are available in literature. 
These estimators are in the form

$$
\hat{\mu}_{\mathrm{SRS}}=\frac{\bar{y}+\beta\left(\mu_{x}-\bar{x}\right)}{(\alpha \bar{x}+\gamma)}\left(\alpha \mu_{x}+\gamma\right)
$$

where $\beta={ }^{\sigma_{x y}} / \sigma_{x}^{2}$

They suggested utilizing some known parameters of the concomitant variable $\mathrm{X}$. where the $\alpha$ and $\gamma$ are the main parameters of interest. Based on known values of coefficient of variation and coefficient of Kurtosis of the auxiliary variable Kadilar and Chingi [1] proposed following modified ratio estimators:

If $\alpha=1$ and $\gamma=V_{x}$, the estimator be :

$$
\hat{\mu}_{\mathrm{SRS} 1}=\frac{\bar{y}+\beta\left(\mu_{x}-\bar{x}\right)}{\left(\bar{x}+V_{x}\right)}\left(\mu_{x}+V_{x}\right)
$$

where $V_{x}$ is the coefficient of variation defined as $V_{x}=$ $\sigma_{x} / \mu_{x}$

If $\alpha=1$ and $\gamma=K_{x}$, then the estimator be :

$$
\hat{\mu}_{\mathrm{SRS} 2}=\frac{\bar{y}+\beta\left(\mu_{x}-\bar{x}\right)}{\left(\bar{x}+K_{x}\right)}\left(\mu_{x}+K_{x}\right)
$$

where $K_{x}$ is the coefficient of Kurtosis defined as $K_{x}=\mu_{x 4} / \mu_{x 2}^{2}$, where $\mu_{x r}=E\left(X-\mu_{x}\right)^{r}$ If $\alpha=K_{x}$ and $\gamma=V_{x}$, then the estimator be :

$$
\hat{\mu}_{\mathrm{SRS} 3}=\frac{\bar{y}+\beta\left(\mu_{x}-\bar{x}\right)}{\left(K_{x} \bar{x}+V_{x}\right)}\left(K_{x} \mu_{x}+V_{x}\right)
$$

If $\alpha=V_{x}$ and $\gamma=K_{x}$, then the estimator be :

$$
\hat{\mu}_{\mathrm{SRS} 4}=\frac{\bar{y}+\beta\left(\mu_{x}-\bar{x}\right)}{\left(V_{x} \bar{x}+K_{x}\right)}\left(V_{x} \mu_{x}+K_{x}\right)
$$

which can the general form as

$$
\bar{y}_{R i}=\frac{\bar{y}+\beta\left(\mu_{x}-\bar{x}\right)}{(\alpha \bar{x}+\gamma)}\left(\alpha \mu_{x}+\gamma\right)
$$

for $\mathrm{i}=1,2,3,4$

The mean square error (MSE) of the above estimators are approximately

$$
\begin{gathered}
\left(\operatorname{MSE}\left(\bar{y}_{R}\right) \cong \frac{1-f}{n}\left[R_{i}^{2} \sigma_{x}^{2}+\sigma_{y}^{2} 1-\rho^{2}\right]\right. \\
\cong \operatorname{MSE}\left(\bar{y}_{R i}\right)=\theta \bar{Y}^{2}\left\lfloor C_{y}^{2}+D_{i}^{2} C_{X}^{2}-2 D_{i} \rho C_{y} C_{x}\right\rfloor
\end{gathered}
$$

for $\mathrm{i}=1,2,3,4$

Where

$$
\begin{gathered}
\mathrm{D}_{1}=\frac{\mu_{y}}{\mu_{x}+V_{x}}, \mathrm{D}_{2}=\frac{\mu_{y}}{\mu_{x}+K_{x}}, \mathrm{D}_{3}=\frac{\mu_{y K_{x}}}{\mu_{x} K_{x}+V_{x}}, \text { and } \mathrm{D}_{4}=\frac{\mu_{y} V_{x}}{V_{x} \mu_{x}+K_{x}} \\
C_{y}=\frac{S_{y}}{\bar{Y}}, C_{x}=\frac{S_{X}}{X}, \rho=\frac{S_{y x}}{S_{y} S_{x}}, \theta=\frac{1}{n} \text { (on ignoring, } f= \\
S_{y}^{2}=\frac{\sum_{i=1}^{N}\left(y_{i}-\bar{Y}\right)^{2}}{N-1}, \\
S_{x}^{2}=\frac{\sum_{i=1}^{N}\left(x_{i}-\bar{X}\right)^{2}}{N-1}, \\
S_{y x}=\frac{\sum\left(y_{i}-\bar{Y}\right)\left(x_{i}-\bar{X}\right)}{N-1}
\end{gathered}
$$

\section{Ratio Estimation under Rank Set Sampling}

Samawi and Muttlak[3]proposed modified ratio estimators in case of rank set sampling. The procedure of ratio estimation under rank set sampling given by Samawi and Muttlak[3]are as under:

Let $Y$ be the variable if interest and $X$ be a suitable concomitant variable which is correlated to $\mathrm{Y}$ and easy to rank. The summary of the RSS procedure is then as following:

1. Select randomly $m^{2}$ bivariate units $(X, Y)$ from the population.

2. Allocate the chosen units into $m$ sets each if size $m$.

3. From the first set, the smallest $X$ and the associated $\mathrm{Y}$ are measured. From the second set, the second smallest of $X$ and the associated $Y$ are measures, We continue in this way until the last set where the largest $\mathrm{X}$ and the associated $\mathrm{Y}$ are measured.

4. Repeat the steps above $r$ times until getting the required number if elements

The associated variable $Y$ is then with error unless the relation between $X$ and $Y$ is perfect. Let us denote $\left(X_{j(i)}, Y_{j[i]}\right)$ as the pair of the $i^{\text {th }}$ order statistics of $X$ and the associated element $Y$ in the $J^{\text {th }}$ cycle. Then the ranked set sample is

$$
\begin{aligned}
& \left(X_{1(1)}, Y_{1[1]}\right), \ldots,\left(X_{1(\mathrm{~m})}, Y_{1[\mathrm{~m}]}\right), \\
& \left(X_{2(1)}, Y_{2[1]}\right), \ldots,\left(X_{2(\mathrm{~m})}, Y_{2[\mathrm{~m}]}\right),
\end{aligned}
$$

$$
\left(\mathrm{X}_{\mathrm{r}(1)}, \mathrm{Y}_{\mathrm{r}[1]}\right), \ldots,\left(\mathrm{X}_{\mathrm{r}(\mathrm{m})}, \mathrm{Y}_{\mathrm{r}[\mathrm{m}]}\right)
$$

Then we define the sample means based on RSS by $\bar{X}^{*}=(1 / \mathrm{n}) \sum_{i=1}^{n} X_{(i)}$ and $\bar{Y}^{*}=(1 / \mathrm{n}) \sum_{i=1}^{n} Y_{[i]}$ with variances are

$$
\operatorname{Var}\left(\bar{X}^{*}\right)=\frac{\sigma_{x}^{2}}{m}-\frac{1}{m^{2}} \sum_{i=1}^{m}\left(\mu_{x(i)}-\mu_{x}\right)^{2},
$$

$\operatorname{Var}\left(\bar{Y}^{*}\right)=\frac{\sigma_{y}^{2}}{m}-\frac{1}{m^{2}} \sum_{i=1}^{m}\left(\mu_{y[i]}-\mu_{y}\right)^{2}$ and

$\operatorname{Cov}\left(\bar{X}^{*}, \bar{Y}^{*}\right)=(1 / \mathrm{m}) \sigma_{x y}-\left(1 / m^{2}\right) \sum_{i=1}^{m} k_{x y[i]}$ with

$$
k_{x y[i]}=\left(\mu_{x(i)}-\mu_{x}\right)\left(\mu_{y[i]}-\mu_{y}\right)
$$

$\hat{R}=\bar{y}^{*} / \bar{x}^{*}$ where $\bar{x}^{*}=\frac{1}{m r} \sum_{k=1}^{r} \sum_{i=1}^{m} X_{k(i)}$ and

$$
\bar{y}^{*}=\frac{1}{m r} \sum_{k=1}^{r} \sum_{i=1}^{m} Y_{k(i)}
$$

and the ratio estimator of the population mean of $\mathrm{Y}$ is

$$
\begin{gathered}
\bar{y}_{R S S}=\bar{y}^{*}\left(\frac{\bar{X}}{\bar{x}^{*}}\right) \\
\operatorname{MSE}\left(\bar{y}_{R s S}\right)=\theta \bar{Y}^{2}\left\lfloor C_{y}^{2}+C_{X}^{2}-2 \rho C_{y} C_{x}\right\rfloor-\left\{Z_{y(i)}-Z_{x(i)}\right\}^{2} \\
\theta=\frac{1}{m r} \\
C_{y}^{2}=\frac{S_{y}^{2}}{\bar{Y}^{2}} \\
C_{x}^{2}=\frac{S_{x}^{2}}{\bar{X}^{2}} \\
C_{y x}=\frac{S_{y x}}{\bar{X} \bar{Y}}=\rho C_{y} C_{x},
\end{gathered}
$$




$$
\begin{gathered}
Z_{x(i)}^{2}=\frac{1}{m^{2} r} \frac{1}{\bar{X}^{2}} \sum_{i=1}^{m} k_{x(i)}^{2}, \\
Z_{y(i)}^{2}=\frac{1}{m^{2} r} \frac{1}{\bar{X}^{2}} \sum_{i=1}^{m} k_{y(i)}^{2}, \\
Z_{y x(i)}=\frac{1}{m^{2} r} \frac{1}{\bar{Y} \bar{X}} \sum_{i=1}^{m} k_{y x(i)} \\
\text { Also } k_{x(i)}=\left(\mu_{x(i)}-\bar{X}\right), k_{y(i)}=\left(\mu_{y(i)}-\bar{Y}\right) \\
k_{y x(i)}=\left(\mu_{x(i)}-\bar{X}\right)\left(\mu_{y(i)}-\bar{Y}\right)
\end{gathered}
$$

Using one degree Taylor series expansion, they showed that this estimator is more efficient than that from SRS with similar form.

\section{Proposed Ratio Estimators under Rank Set Sampling}

In this section we suggest to use similar form of estimators as given by Kadilar and Chingi [1]. We assume that the population mean of the auxiliary variable is known beforehand, we also assume that the relation between $\mathrm{X}$ and $\mathrm{Y}$ is positive and approximately linear.

Based on RSS, we suggest ratio-type estimators for the mean in the form

$\hat{\mu}_{\mathrm{RSS}}=\frac{\bar{y}^{*}+\beta\left(\mu_{x}-\bar{x}^{*}\right)}{\left(\alpha \bar{x}^{*}+\gamma\right)}\left(\alpha \mu_{x}+\gamma\right)$ where $\alpha$ and $\gamma$ are positive constants,

$\operatorname{and} \beta=\sigma_{x y} / \sigma_{x}^{2}$

Let us take some special cases of $\alpha$ and $\gamma$ various combinations of known values of Median, Quartile deviation of auxiliary variable in case of RSS.

CASE-1: If the Quartile Deviation and Median of the concomitant variable are available, we may choose this parameter to be values for $\sigma$ and $\gamma$ in the estimator above. For examples:

If $\alpha=1$ and $\gamma=Q d$, then we have the estimator :

$$
\hat{\mu}_{\mathrm{RSS} 1}=\frac{\bar{y}^{*}+\beta\left(\mu_{x}-\bar{x}^{*}\right)}{\left(\bar{x}^{*}+Q d\right)}\left(\mu_{x}+Q d\right)
$$

If $\alpha=1$ and $\gamma=M d$, then we have the estimator

$$
\hat{\mu}_{\mathrm{RSS} 2}=\frac{\bar{y}^{*}+\beta\left(\mu_{x}-\bar{x}^{*}\right)}{\left(\bar{x}^{*}+M d\right)}\left(\mu_{x}+M d\right)
$$

If $\alpha=M d$ and $\gamma=Q d$, then we have the estimator :

$$
\hat{\mu}_{\mathrm{RSS} 3}=\frac{\bar{y}^{*}+\beta\left(\mu_{x}-\bar{x}^{*}\right)}{\left(M d \bar{x}^{*}+Q d\right)}\left(M d \mu_{x}+Q d\right)
$$

If $\alpha=Q d$ and $\gamma=M d$, then we have the estimator :

$$
\hat{\mu}_{\mathrm{RSS} 4}=\frac{\bar{y}^{*}+\beta\left(\mu_{x}-\bar{x}^{*}\right)}{\left(Q d \bar{x}^{*}+M d\right)}\left(Q d \mu_{x}+M d\right)
$$

As we know, $\bar{y}_{R s s}=\bar{y}^{*}\left(\frac{\bar{x}}{\bar{x}^{*}}\right)$ also we can denote $\bar{y}_{R s s}$ as $\hat{\mu}_{R s s}$

Where

$$
\bar{x}^{*}=\frac{1}{m r} \sum_{k=1}^{r} \sum_{i=1}^{m} X_{k(i)},
$$

$$
\bar{y}^{*}=\frac{1}{m r} \sum_{k=1}^{r} \sum_{i=1}^{m} Y_{k(i)}
$$

Then

$$
\bar{y}_{R s s i}=\frac{\bar{y}^{*}+\beta\left(\mu_{x}-\bar{x}^{*}\right)}{\left(\alpha \bar{x}^{*}+\gamma\right)}\left(\alpha \mu_{x}+\gamma\right)(\text { for } \mathrm{i}=1,2,3,4)
$$

The bias and mean square error of the above estimator based on RSS will be:

$$
\begin{gathered}
\operatorname{Bias}\left(\bar{y}_{R s s i}\right)=\bar{Y}\left[\theta\left(G_{i}^{2} C_{x}^{2}-G_{i} \rho C_{y} C_{x}\right)\right. \\
\left.-\left\{G_{i}^{2} Z_{x(i)}^{2}-G_{i} Z_{y x(i)}\right\}\right] \\
\operatorname{MSE}\left(\bar{y}_{R s s i}\right)=\bar{Y}^{2}\left[\theta\left\{C_{y}^{2}+G_{i}^{2} C_{x}^{2}-2 G_{i} \rho C_{y} C_{x}\right\}\right. \\
\left.-\left\{Z_{y(i)}-G_{i} Z_{x(i)}\right\}^{2}\right]
\end{gathered}
$$

Where $G_{i}=\frac{\bar{Y} \alpha}{\alpha \bar{X}+\gamma}$

Where $\alpha$ and $\gamma$ can be replaced by various linear combinations of known values of Median, quartile deviation, of the auxiliary variable which is mentioned in the above case.

\section{Bias and Mean Square Estimation of Proposed Estimators}

Since the bias and mean square error of the above estimator based on RSS will be :

$$
\begin{gathered}
\operatorname{Bias}\left(\bar{y}_{R s s i}\right)=\bar{Y}\left[\theta\left(G_{i}^{2} C_{x}^{2}-G_{i} \rho C_{y} C_{x}\right)\right. \\
\left.-\left\{G_{i}^{2} Z_{x(i)}^{2}-G_{i} Z_{y x(i)}\right\}\right] \\
\operatorname{MSE}\left(\bar{y}_{R s s i}\right)=\bar{Y}^{2}\left[\theta\left\{C_{y}^{2}+G_{i}^{2} C_{x}^{2}-2 G_{i} \rho C_{y} C_{x}\right\}\right. \\
\left.-\left\{Z_{y(i)}-G_{i} Z_{x(i)}\right\}^{2}\right]
\end{gathered}
$$

Where $G_{i}=\frac{\bar{Y} \alpha}{\alpha \bar{X}+\gamma}$

To obtain the bias and MSE of $\bar{y}_{R s S i}$

We put $\bar{y}^{*}=\bar{Y}\left(1+\varepsilon_{0}\right)$

And $\bar{x}^{*}=\bar{X}\left(1+\varepsilon_{1}\right)$, so that the expectation of $\varepsilon_{0}$ and $\varepsilon_{1}$ is equal to zero

$$
\begin{gathered}
E\left(\varepsilon_{0}\right)=E\left(\varepsilon_{1}\right)=0 \\
V\left(\varepsilon_{0}\right)=E\left(\varepsilon_{0}^{2}\right)=\frac{V\left(\bar{y}^{*}\right)}{\bar{Y}^{2}} \\
=\frac{1}{m r} \frac{1}{\bar{Y}^{2}}\left[\frac{1}{m} \sum_{i=1}^{m} k_{y(i)}^{2}\right]=\left\lfloor\theta C_{y}^{2}-Z_{y(i)}^{2}\right\rfloor
\end{gathered}
$$

Similarly

$$
V\left(\varepsilon_{1}\right)=E\left(\varepsilon_{1}^{2}\right)=\left\lfloor\theta C_{x}^{2}-Z_{x(i)}^{2}\right\rfloor
$$

And

$$
\begin{gathered}
\operatorname{Cov}\left(\varepsilon_{0}, \varepsilon_{1}\right)=E\left(\varepsilon_{0}, \varepsilon_{1}\right)=\frac{\operatorname{Cov}\left(\bar{y}^{*} \bar{x}^{*}\right)}{\bar{Y} \bar{X}} \\
=\frac{1}{\bar{Y} \bar{X}} \frac{1}{m r}\left[S_{y x}-\frac{1}{m} \sum_{i=1}^{m} k_{y x(i)}\right]=\left[\theta \rho C_{y} C_{x}-Z_{y x(i)}\right]
\end{gathered}
$$

Where 


$$
\begin{gathered}
\theta=\frac{1}{m r^{\prime}} \\
C_{y}^{2}=\frac{S_{y}^{2}}{\bar{Y}^{2}} \\
C_{x}^{2}=\frac{S_{x}^{2}}{\bar{X}^{2}} \\
C_{y x}=\frac{S_{y x}}{\bar{X} \bar{Y}}=\rho C_{y} C_{x}, \\
Z_{x(i)}^{2}=\frac{1}{m^{2} r} \frac{1}{\bar{X}^{2}} \sum_{i=1}^{m} k_{x(i)}^{2}, \\
Z_{y(i)}^{2}=\frac{1}{m^{2} r} \frac{1}{\bar{X}^{2}} \sum_{i=1}^{m} k_{y(i)}^{2}, \\
Z_{y x(i)}=\frac{1}{m^{2} r} \frac{1}{\bar{Y} \bar{X}} \sum_{i=1}^{m} k_{y x(i)}
\end{gathered}
$$

Also $k_{x(i)}=\left(\mu_{x(i)}-\bar{X}\right), k_{y(i)}=\left(\mu_{y(i)}-\bar{Y}\right)$

and

$$
k_{y x(i)}=\left(\mu_{x(i)}-\bar{X}\right)\left(\mu_{y(i)}-\bar{Y}\right)
$$

Proof :

BIAS

$$
\operatorname{Bias}\left(\bar{y}_{R s s i}\right)=E\left(\bar{y}_{R s s i}-\bar{Y}\right)
$$

Here $\bar{y}_{R s s i}=\bar{Y}\left(1+\varepsilon_{0}\right)\left(1+G_{i} \varepsilon_{1}\right)^{-1}$

Suppose $\left(G_{i} \varepsilon_{1}\right)<1$ so that $\left(1+G_{i} \varepsilon_{1}\right)^{-1}$ is expandable

$$
\bar{y}_{R s s i}=\bar{Y}\left(1+\varepsilon_{0}\right)\left\{1-G_{i} \varepsilon_{1}+G_{i}^{2} \varepsilon_{1}^{2}+O\left(G_{1} \varepsilon_{1}\right)\right\}
$$

(Using Taylor series expansion, where $O\left(\varepsilon_{1}\right)$ with power more than 2 are neglected for large power of $\varepsilon_{1}$.

$$
\begin{gathered}
\operatorname{Bias}\left(\bar{y}_{R s s i}\right)=\bar{Y}\left[G_{i}^{2} E\left(\varepsilon_{1}^{2}\right)-G_{i} E\left(\varepsilon_{0} \varepsilon_{1}\right)\right] \\
=\bar{Y}\left[G_{i}^{2}\left(\theta C_{x}^{2}-Z_{x(i)}^{2}\right)-G_{i}\left(\theta \rho C_{y} C_{x}-Z_{y x(i)}\right)\right] \\
\Rightarrow \gg \operatorname{Bias}\left(\bar{y}_{R s s i}\right)=\bar{Y}\left[\theta\left(G_{i}^{2} C_{x}^{2}-G_{i} \rho C_{y} C_{x}\right)-\left\{G_{i}^{2} Z_{x(i)}^{2}\right.\right. \\
\left.\left.\left.-G_{i} Z_{y x(i)}\right\}\right)\right]
\end{gathered}
$$

Proof

MEAN SQUARE ERROR:

$$
\begin{gathered}
\operatorname{MSE}\left(\bar{y}_{R s s i}\right)=E\left(\bar{y}_{R s s i}-\bar{Y}\right)^{2} \\
=\bar{Y}^{2} E\left[\varepsilon_{0}-G_{i} \varepsilon_{1}+G_{i}^{2} \varepsilon_{1}^{2}-2 G_{i} \varepsilon_{0} \varepsilon_{1}\right]^{2} \\
=\bar{Y}^{2} E\left[\varepsilon_{0}^{2}+G_{i}^{2} \varepsilon_{1}^{2}-2 G_{i} \varepsilon_{0} \varepsilon_{1}\right] \\
=\bar{Y}^{2}\left[\theta C_{y}^{2}-Z_{y(i)}^{2}+G_{i}^{2}\left(\theta C_{x}^{2}-Z_{x(i)}^{2}\right)\right. \\
\left.-2 G_{i}\left(\theta \rho C_{y} C_{x}-Z_{y x(i)}\right)\right] \\
=\bar{Y}^{2}\left[\theta\left\{C_{y}^{2}+G_{i}^{2} C_{x}^{2}-2 G_{i} \rho C_{y} C_{x}\right\}-\left\{Z_{y(i)}^{2}+G_{i}^{2} Z_{x(i)}^{2}\right.\right. \\
\left.\left.-2 G_{i} Z_{y x(i)}\right\}\right] \\
=\gg \operatorname{MSE}\left(\bar{y}_{R s s i}\right)=\bar{Y}^{2}\left[\theta\left\{C_{y}^{2}+G_{i}^{2} C_{x}^{2}-2 G_{i} \rho C_{y} C_{x}\right\}\right. \\
\left.-\left\{Z_{y(i)}-G_{i} Z_{x(i)}\right\}^{2}\right]
\end{gathered}
$$

\section{Efficiency Comparison and Numerical Illustration}

The behaviour of the above estimators is studied and compared with the corresponding estimators from SRS. Let us assume that the variable of interest $Y$ and a concomitant variable $X$ are correlated with a correlation coefficient $\rho$. Assume also that $X$ and $Y$ have a bivariate normal

\begin{tabular}{|c|c|c|c|c|c|}
\hline$\rho$ & $\mathrm{m}$ & $E f_{1}$ & $E \boldsymbol{f}_{2}$ & $E f_{3}$ & $E f_{4}$ \\
\hline \multirow{4}{*}{0.99} & 2 & 1.89 & 1.86 & 1.74 & 1.42 \\
\hline & 4 & 2.55 & 2.34 & 1.79 & 1.49 \\
\hline & 6 & 2.82 & 2.64 & 1.88 & 1.52 \\
\hline & 12 & 3.92 & 3.13 & 2.1 & 1.71 \\
\hline \multirow{4}{*}{0.88} & 2 & 1.43 & 1.39 & 1.44 & 1.12 \\
\hline & 4 & 1.84 & 1.74 & 1.85 & 1.14 \\
\hline & 6 & 1.91 & 1.76 & 1.91 & 1.23 \\
\hline & 12 & 2.24 & 2.01 & 2.24 & 1.35 \\
\hline \multirow{4}{*}{0.77} & 2 & 1.18 & 1.16 & 1.22 & 1.04 \\
\hline & 4 & 1.41 & 1.32 & 1.41 & 1.13 \\
\hline & 6 & 1.49 & 1.38 & 1.49 & 1.26 \\
\hline & 12 & 1.58 & 1.39 & 1.58 & 1.38 \\
\hline
\end{tabular}
distribution with parameter. Assume that $(\mathrm{X}, \mathrm{Y})$ follow a bivariate normal distribution, the performance of simple regression using BVSRS,BVSYS and BVRSS is judged with the help of a data set. The original data were collected on two variables of Pinus wllichiana: $X$, the diameter in centimeters at breast height and $Y$, the entire height in meters .Thepopulationconsistsof 275 trees. Thesummarystatis ticsofthedataarereportedinTable-1.

Table 1. Summary statistics of the Pinus data

\begin{tabular}{ccc}
\hline & DBH $(\mathrm{cm})$ & Height $(\mathrm{m})$ \\
\hline Mean & 21.44 & 15.66 \\
Standard Deviation & 20.95 & 17.06 \\
Range & 216.80 & 70.87 \\
Minimum & 2.20 & 0.90 \\
Maximum & 219 & 71.77 \\
Count & 275 & 275 \\
\hline
\end{tabular}

Using the real data set of PinusWallichiana taken from Langate block of district Baramulla from Kashmir valley data set, different sample where generated using SRS and RSS from a bivariate normal distribution using the above combinations of $\alpha$ and $\gamma$. From this distribution we generated 440 samples based on RSS with 10 cycles and another 440 samples using SRS. For each sample, the mean square errors are computed respectively. The above bivariate normal distribution was generated in R-Software using the function mvtnormfrom library(mvtnorm). Different values of $\rho$ and $m$ are used and the results are shown in Tables- 2 and 3 .

Table 2. Linear combination of Quartile deviation and Median 
Table 3. Linear combination of Quartile deviation and Coefficient of Skewness

\begin{tabular}{|c|c|c|c|c|c|}
\hline$\rho$ & $\mathrm{m}$ & $E f_{1}$ & $\boldsymbol{E} \boldsymbol{f}_{2}$ & $E \boldsymbol{f}_{3}$ & $E \boldsymbol{f}_{4}$ \\
\hline \multirow{4}{*}{0.66} & 2 & 2.09 & 1.97 & 1.65 & 2.12 \\
\hline & 4 & 2.57 & 2.02 & 1.72 & 2.78 \\
\hline & 6 & 2.87 & 2.11 & 1.75 & 3.05 \\
\hline & 12 & 3.36 & 2.33 & 1.94 & 4.15 \\
\hline \multirow{4}{*}{0.55} & 2 & 1.62 & 1.67 & 1.35 & 1.66 \\
\hline & 4 & 1.97 & 2.08 & 1.37 & 2.07 \\
\hline & 6 & 1.99 & 2.14 & 1.46 & 2.14 \\
\hline & 12 & 2.24 & 2.47 & 1.58 & 2.47 \\
\hline \multirow{4}{*}{0.44} & 2 & 1.39 & 1.45 & 1.27 & 1.41 \\
\hline & 4 & 1.55 & 1.64 & 1.36 & 1.64 \\
\hline & 6 & 1.61 & 1.72 & 1.49 & 1.72 \\
\hline & 12 & 1.62 & 1.81 & 1.61 & 1.81 \\
\hline
\end{tabular}

The efficiency of RSS with respect to corresponding estimators of SRS

$E f_{i}=\frac{M S E(\widehat{\mu} \mathrm{SRS})_{i}}{M S E(\widehat{\mu} \mathrm{RSS})_{i}}$, where $(i=1,2,3,4)$

\section{Conclusions}

From the results of Tables- 2 and 3 it is concluded the modified ratio estimators under rank set sampling are more efficient than the classical ratio estimators given by Kadilar and Chingi[1] based on simple random sampling, also efficiency of RSS estimators decreases as the correlation coefficient decreases and efficiency is increasing as the set size $m$ is increasing. The above results occurred because rank set samples are more regularly spaced than those obtained from SRS, therefore more representative of the population. Because of ranking the RSS procedure induces stratification at sample level which involves the gained precision in this scheme.

\section{Acknowledgments}

The first author wishes to record his gratitude and thanks to University Grants Commission, Govt of India, for providing the UGC National Fellowship for perusing the Doctorate programme in Statistics.

\section{REFERENCES}

[1] Kadilar,C. and Cingi,H.(2004).Ratio estimateors in simple random sampling, Applied Mathematics and Computation151,893-902.

[2] McIntyre, G. A. (1952) A Method of Unbiased Selective Sampling Using Ranked Set. Australian, J. Agricultural Research, 3, 385-390.

[3] Samawi, H. M. and Muttlak, H. A. (1996) Estimation of Ratio Using Rank Set Sampling, Biom. Journal,38, 753-764.

[4] W.G.Cochran,(1977).Sampling Techniques, Third Edition, Wiley Eastern Limited.

[5] Murthy,M.N.(1967).Sampling theory and methods, Statistical Publishing Society, Calcutta, India.

[6] Prasad,B.(1989) Some improved ratio type estimators of population mean and ratio infinite population sample surveys, Communications in Statistics: Theory and Methods18,379-392.

[7] Sen,A.R.(1993):Some early development sin ratio estimation, Biometric Journal 35(1),3-13.

[8] Singh,H.P. and Tailor,R.(2003).Use of known correlation co-efficient in estimating the finite population means, Statistics inTransition6(4), 555-560.

[9] Singh,H.P. and Tailor,R.(2005).Estimation of finite population mean with known co-efficient of variation of anauxiliary, STATISTICA,annoLXV,n.3,pp301-313

[10] Singh,H.P.,Tailor,R.,Tailor,R. and Kakran,M.S.(2004). An Improved Estimator of population mean using Power transformation, Journal of the Indian Society of Agricultural Statistics 58(2),223-230.

[11] Kadilar,C. and Cingi,H.(2006).An Improvement in Estimating the Population mean by using the Correlation Co-efficient, Hacettepe Journal of Mathematics and Statistics Volume 35(1),103-109

[12] Koyuncu,N.and Kadilar,C.(2009).Efficient Estimators for the Population mean, Hacettepe Journal of Mathematics and Statistics, Volume 38(2),217-225.

[13] Yan,Z.andTian,B.(2010).RatioMethodtotheMeanEstimation UsingCo-efficientofSkewnessofAuxiliary Variable,ICICA2010,PartII,CCIS106,pp.103-110.

[14] M.Iqbal, S.Maqbool,S.A.Mir, Imran .Khan, Nageena.Nazirand Fehim.Jeelani. (2013).Modified Ratio Estimators of Population Mean Using Linear Combination of Co-efficient of Kurtosis and Quartile Deviation. International Journal of Modern Mathematical Sciences, USA. Florida., 8(3): 149-15. 